Bio - grafía. Escritos sobre la Biología y su Enseñanza. ISSN 2027-1034

Edición Extraordinaria. p.p. 502 - 508

Memorias del IX Encuentro Nacional de Experiencias en Enseñanza de la Biología y la Educación Ambiental. IV Congreso Nacional de Investigación en Enseñanza de la Biología.

\title{
LA UVE HEURÍSTICA DE GOWIN COMO ESTRATEGIA DIDÁCTICA PARA LA ENSEÑANZA Y EL APRENDIZAJE DE LAS CIENCIAS NATURALES Y EDUCACIÓN AMBIENTAL EN LA INSTITUCIÓN EDUCATIVA JOSÉ ANTONIO GALÁN DE SAN PELAYO-CORDOBA
}

\author{
GOWIN'S HEURISTIC UVE AS A DID ACTIC STRATEGY FOR THE TEACHING AND \\ LEARNING OF NATURAL SCIENCES AND ENVIRONMENT AL EDUCATION TO \\ STUDENTS IN THE EDUCATIONAL INSTITUTION JOSÉ ANTONIO GALÁN DE SAN \\ PELAYO-CORDOBA
}

\author{
Mary Luz Doria Rojas \\ Lina Marcela Lozano Conde
}

\section{RESUMEN}

La presente investigación implementó la UVE Heurística de Bob Gowin, buscaba saber las estrategias utilizadas por los docentes en la enseñanza de la ciencias naturales y educación ambiental e implementar la UVE para generar aprendizaje, La metodología implementada correspondió a un tipo de investigación cualitativa con un enfoque fenomenológico trascendental, desarrollándose en tres fases de cinco sesiones de trabajo donde se implementó ésta a partir de las temáticas de bilogía. Los resultados se recolectaron a partir de técnicas e instrumentos como la encuesta, la observación, los talleres de formación, rubricas y entrevista de tipo semiestructurada. Los resultados mostraron la apropiación de la estrategia didáctica, además del desarrollo de habilidades de indagación y construcción del conocimiento para dar respuesta a la pregunta central planteada a los estudiantes durante la intervención con la UVE Heurística de Gowin como estrategia didáctica de aprendizaje.

PALABRAS CLAVES: Aprendizaje, Estrategia didáctica, la UVE H. Gowin.

\section{SUMMARY}

The present research implemented the UVE Heurística of Bob Gowin, it looked for to know the strategies used by the teachers in the education of the natural sciences and environmental education and to implement the UVE to generate learning, The implemented methodology corresponded to a type of qualitative research with a focus phenomenological, developing in three phases of five work sessions where it was implemented from the themes of biology. The results were collected from techniques and instruments such as survey, observation, training workshops, rubrics and semi-structured 
Bio - grafía. Escritos sobre la Biología y su Enseñanza. ISSN 2027-1034

Edición Extraordinaria. p.p. 502 - 508

Memorias del IX Encuentro Nacional de Experiencias en Enseñanza de la Biología y la

Educación Ambiental. IV Congreso Nacional de Investigación en Enseñanza de la Biología.

interview. The results showed the appropriation of the didactic strategy, as well as the development of skills of inquiry and construction of knowledge to answer the central question posed to the students during the intervention with the Gowin Heuristic UVE as didactic learning strategy.

KEY WORDS: Learning, Didactic Strategy, UVE H. Gowin.

\section{INTRODUCCIÓN}

Inicialmente vale la pena resaltar la importancia de la educación en los seres humanos como parte fundamental de su formación. En la actualidad, las Ciencias Naturales y Educación Ambiental han tomado una importancia muy significativa en los procesos de enseñanza y aprendizaje, no solo en las Instituciones Educativas (IE) sino también en la sociedad; ya que esta rama de las ciencias tiene una característica en particular como es el conocimiento de los avances científicos que demandan una permanente búsqueda de conocimiento y estar actualizado con los últimos avances en lo que tienen que ver con los cambios que en materia ambiental que se están presentando, tiene gran influencias en las dinámicas de supervivencia del hombre en el planeta.

En la Conferencia Mundial sobre la Ciencia para el siglo XXI, auspiciada por la UNESCO y el Consejo Internacional para la Ciencia, se concluyó (Declaración de Budapest, 1999) que:

"Para que un país esté en condiciones de atender a las necesidades naturales. La enseñanza y la evaluación de las ciencias naturales. Aportes para la Enseñanza de las fundamentales de su población, la enseñanza de las ciencias y de la tecnología es un imperativo estratégico" (pág. 13)... Una visión social de las ciencias ha sido desarrollada también por diversos autores, como B. Macedo y colaboradores (2006), quienes señalan que los beneficios de la ciencia y de la tecnología se traducen en inequidad e injusticia por su desigual distribución, tanto entre países como dentro de éstos, existiendo grupos excluidos del conocimiento científico y sus beneficios. Plantean que la ciencia y la tecnología no solo deben mejorar las condiciones de quienes viven en situaciones de pobreza, sino que los avances científicos deben ser bien utilizados ... compromiso ético de disminuir la exclusión y terminar con la concentración del conocimiento, que significa concentración del poder (2016. pág. 3)

Es necesario el uso de estrategias para la enseñanza de esta ciencia, además poder implementar la UVE para generar en los estudiantes la apropiación de ésta y posterior uso en el desarrollo académico desde la autonomía que le brinda frente a la búsqueda del conocimiento y la convierte en una de las principales estrategias para generar aprendizaje significativo en estudiantes.

Como lo señalan los autores Valencia, Cardona y Mestra, et al., (1999) donde exponen la relación de Vygotsky \& Piaget: 
Bio - grafía. Escritos sobre la Biología y su Enseñanza. ISSN 2027-1034

Edición Extraordinaria. p.p. 502 - 508

Memorias del IX Encuentro Nacional de Experiencias en Enseñanza de la Biología y la

Educación Ambiental. IV Congreso Nacional de Investigación en Enseñanza de la Biología.

Como se ha demostrado la concepción Vygotskyana sobre las relaciones en el desarrollo cognitivo y aprendizaje difieren en buena medida a la piagetiana. Mientras Piaget sostiene que lo que un niño pueda aprender está determinado por su nivel de desarrollo cognitivo, Vygotsky piensa que este último es el que está condicionado por el aprendizaje. (p.32)

Por consiguiente, frente a las dificultades entorno a la enseñanza y aprendizaje de las ciencias naturales y educación ambiental, y la desmotivación que existe entre algunos docentes y estudiantes por implementar estrategias para el acceso al conocimiento, se han desarrollado diversos tipos frente a estas dificultades para el desarrollo de capacidades, por lo cual, existe la necesidad de implementar la UVE Heurística de Bob Gowin, "fue diseñada para ayudar a los estudiantes y profesores a captar el significado de los materiales que se van a aprender" Novak, J. y Gowin, B. (1988). Se implementó en la búsqueda de generar saber y procesos de aprendizaje duraderos, se ha venido implementando por medio de programas educativos en países desarrollados. Como lo indican Herrera y Sánchez (2012) donde afirman que:

Esta propuesta metodológica no es una "novedad" en la enseñanza de las ciencias: desde hace ya un par de décadas se desarrollan programas en Francia y Estados Unidos, y en otros países del mundo. Así, desde mediados de la década de los 80, tanto la Academia de Ciencias de Francia como el Centro Nacional de Recursos Científicos de Estados Unidos han desarrollado programas para el mejoramiento de la enseñanza de las ciencias a nivel de las escuelas (p.104).

Es necesario enfocarnos e investigar y aplicar estrategias de aprendizaje que involucren tanto al maestro como al alumno, existen escuelas que realizan las estrategias pero el alumno no es conocedor de qué tipo de estrategia utiliza el maestro, en este sentido, Díaz y Molina (2010) indican que el hay carencia en los estudiantes de destrezas para maximizar el potencial cognitivo, ello evidenciado en la falta de vocabulario, habilidades de lectoescritura, matemáticas, disciplina de estudio, actitud positiva por el aprendizaje y alcanzar su formación intelectual (p.33)

Es importante destacar la necesidad que tuvo la implementación de la UVE Heurística como estrategia didáctica para el aprendizaje, buscando la motivación e interés de los estudiantes por aprender a partir de la indagación que exige el resolver la pregunta central de ésta, siendo el pretexto para que ellos realicen de manera autónoma la solución y apropiación, no solo de la UVE, sino también del conocimiento indagado.

\section{METODOLOGÍA}

Para la realización de este estudio se optó por la aplicación de un tipo de investigación cualitativa con un diseño fenomenológico trascendental, el cual explica Salgado, A. (2007) que este se enfoca en "las experiencias individuales subjetivas de los participantes y también responde a la pregunta ¿Cuál es el significado, estructura y esencia de una experiencia vivida por un individuo, un grupo o sociedad al respecto de un fenómeno?" ( $\mathrm{p}$. 73). Esta tuvo como finalidad resolver problemas cotidianos e inmediatos, que 
Bio - grafía. Escritos sobre la Biología y su Enseñanza. ISSN 2027-1034

Edición Extraordinaria. p.p. 502 - 508

Memorias del IX Encuentro Nacional de Experiencias en Enseñanza de la Biología y la

Educación Ambiental. IV Congreso Nacional de Investigación en Enseñanza de la Biología.

involucraran al maestro, al alumno y a la estrategia didáctica, formando una triada enfocada con un propósito fundamental que se centró en aportar información que guío a la enseñanza y el aprendizaje de las ciencias naturales de los alumnos grado $9^{\circ} 4$ de la institución educativa José Antonio Galán, desarrollo e implementación de esta investigación se realizaron cinco sesiones. Se utilizaron técnicas para la recolección de la información como fueron las rubricas, observaciones, diario de campo y una entrevista. Permitió evidenciar la fenomenología trascendental como base sólida de la investigación desde la autoevaluación que se hizo al estudiante a partir de la adquisición de conocimiento. Por ello, es indispensable resaltar la importancia que tuvo la implementación del método fenomenológico para conocer la raíz de los problemas académicos de los estudiantes a partir de la aplicación de encuesta, observación entre otros, la aplicación de la UVE como mediadora para la solución del poco interés que tenían por aprender ciencia (biología) y desempeño académico de la asignatura de ésta, también se pudo facilitar el estudio de la muestra, lo cual corrobora Zambrano, (2016) quien señala que:

La fenomenología facilita ir más allá del estudio de los comportamientos observables y controlables, como fenómenos naturales en el positivismo. Por tanto, debe estudiar la vivencia del ser humano en una determinada situación, siempre procurando interrogar la experiencia vivida, los significados que el sujeto le atribuye. Es decir, es una alternativa para la investigación con seres humanos, un camino diferente del método experimental utilizado por las ciencias naturales. (s.p)

La población en la que se realizó la investigación correspondió a 125 estudiantes de la Institución Educativa José Antonio Galán de San Pelayo; la cual cuenta con 125 estudiantes de ciencias naturales entre las dos jornadas académicas para el grado de $9^{\circ}$. Para escoger la muestra se tuvo en cuenta un muestreo no probabilísticos de tipo intencional o de convivencia seleccionando a 25 estudiantes del grado $9^{\circ} 4$ de la jornada de la tarde.

\section{RESULTADOS Y DISCUSIÓN}

Respecto al aprendizaje generado por la implementación de la estrategia didáctica UVE H. GOWIN a partir de la implementación de la realización de sesiones de trabajo, se observó durante los talleres que es una ayuda académica que le ha dado aportes al conocimiento de la muestra de estudio. Todo esto se simplificó y permitió generar un impacto en la participación en el aula donde los jóvenes comunicaron su opinión sobre la UVE Heurística, dando un resultado en su aplicabilidad y generación de conocimiento recomendándola para su uso estratégico. Del tal forma, que si hubo aprendizaje, se empoderaron de la estrategia y la docente a cargo de la asignatura de biología fue visualizador de las exposiciones y también los evaluó con notas apreciativa alcanzando así una mejora académica evidenciada en la apropiación (actividades en clase) que los estudiantes demostraron en el abordaje de los temas a través de la UVE. 
Bio - grafía. Escritos sobre la Biología y su Enseñanza. ISSN 2027-1034

Edición Extraordinaria. p.p. 502 - 508

Memorias del IX Encuentro Nacional de Experiencias en Enseñanza de la Biología y la

Educación Ambiental. IV Congreso Nacional de Investigación en Enseñanza de la Biología.

\section{CONCLUSIONES}

Los estudiantes desarrollaron habilidades de indagación e interpretación, obteniendo de esta forma que en su autoevaluación respondieran a los sucesos con afirmaciones de valor en los conocimientos estudiados.

Durante los acontecimientos o fenómenos a estudiar los estudiantes se enfocaron en todo lo que constituía la temática de este periodo la cual fue la genética, en donde se plantearon preguntas problematizadora a las cuales les dieron respuesta con una buena implementación y apropiación de la UVE Heurística.

Fue evidente el desarrollo de habilidades de indagación científica y la construcción del conocimiento para dar respuesta a la pregunta central planteada, demostrando apropiación de la estrategia didáctica. Las preguntas planteadas estaban relacionadas con los ejes temáticos trabajados.

Las experiencias de indagación de los estudiantes a partir de la formulación de preguntas científicas y la respuesta de éstas con el uso de la UVE Heurística, como lo indica Ávila, Martínez, Monterrosa, et al., (1999) señalando que " Necesitamos de una educación que tenga como fin la integración dinámica de la experiencia personal y como método la participación activa del educando en la búsqueda de su propio y autentico aprendizaje" (p.20) siendo este es la huella dejada por los pensamientos, es por eso que aprendemos pensando y la calidad del resultado de ese aprendizaje viene determinada por la calidad de la construcción de nuestros pensamientos.

Se observó apropiación en el buen uso del dominio metodológico y conceptual para darle respuestas a la pregunta central con base a cada una de las temáticas abordadas en los encuentros al implementar la estrategia didáctica, los estudiantes buscaban aprender de forma organizada, grupal, detallada y sintética, logrando el desarrollo de habilidades de indagación científica y la construcción del conocimiento.

\section{BIBLIOGRAFÍA}

Ávila, R; Martínez, G; Monterrosa, I; Ramos, N y Rosso, A. (1999). Enfoque integrador en la enseñanza y aprendizaje. Tesis de especialización en didáctica de las Ciencias Naturales. Universidad de Córdoba. Montería, Colombia.

Castro, M. Gutiérrez, E. Marín, M. et al., (2015). Impacto de la uve de Gowin en el desarrollo de conocimientos, razonamientos e inteligencias múltiples. Tesis de pregrado. Universidad Juárez Autónoma de Tabasco. Villahermosa, México. Tomado el 5 de febrero del 2017 de: http://www.revistas.ujat.mx/index.php/perspectivas/article/view/1196/971 
Bio - grafía. Escritos sobre la Biología y su Enseñanza. ISSN 2027-1034

Edición Extraordinaria. p.p. 502 - 508

Memorias del IX Encuentro Nacional de Experiencias en Enseñanza de la Biología y la

Educación Ambiental. IV Congreso Nacional de Investigación en Enseñanza de la Biología.

Chrobak, R. \& Prieto, A. (2010). La herramienta UVE del conocimiento para favorecer la creatividad de docentes y estudiantes. Anales de Psicología, Murcia, España.

Coral, E \& García, J. (2014) la v heurística como herramienta metodológica en el aprendizaje significativo de las ciencias naturales. Trabajo de investigación. Escuela Popular Champagnat, Sede Jongovito. Pasto, Colombia. Tomado el 04 de marzo del 2017 de: http://biblioteca.udenar.edu.co:8085/atenea/biblioteca/90545.pdf

Escatell, M. (2016). Diagrama V de Gowin. Recuperado el 8 de febrero de 2017 de: https://www.youtube.com/watch?v=uD3hjvFBt44

Fernández, G (2014) Aplicación de la V de Gowin al método de proyectos: Una propuesta de aprendizaje significativo. Trabajo de Máster. Centro Universitario de Mérida. España. Recuperado el 20 de abril del 2017 de: http://es.calameo.com/read/002932346e5f69101bde0

Herrera, E. \& Sánchez, I. (2012). La uve de Gowin como instrumento de aprendizaje y evaluación de habilidades de indagación en la unidad de fuerza y movimiento. Tesis de doctorado. Universidad Bio-Bio, octava región Chile.

Morantes, Z; Arrieta, X. \& Nava, M. (2013) La v de Gowin como mediadora en el desarrollo de la información investigativa. Góndola. Volumen 8 (2). Venezuela. Extraído el 23 de enero del 2017 de: http://revistas.udistrital.edu.co/ojs/index.php/GDLA/article/view/5147/9352

UNESCO (2016). Aportes para la Enseñanza de Ciencias Naturales. Este informe ha sido elaborado por MIDE UC por encargo de la Oficina Regional de Educación para América Latina y el Caribe, OREALC/UNESCO Santiago Naciones Unidas, Asamblea General. Publicado en 2016 por la Organización de las Naciones Unidas para la Educación, la Ciencia y la Cultura, 7, place de Fontenoy, 75352 París 07 SP, Francia y la Oficina Regional de Educación de la UNESCO para América Latina y el Caribe, OREALC/UNESCO Santiago. http://unesdoc.unesco.org/images/0024/002447/244733s.pdf 
Bio - grafía. Escritos sobre la Biología y su Enseñanza. ISSN 2027-1034

Edición Extraordinaria. p.p. 502 - 508

Memorias del IX Encuentro Nacional de Experiencias en Enseñanza de la Biología y la

Educación Ambiental. IV Congreso Nacional de Investigación en Enseñanza de la Biología.

\section{ANEXOS:}
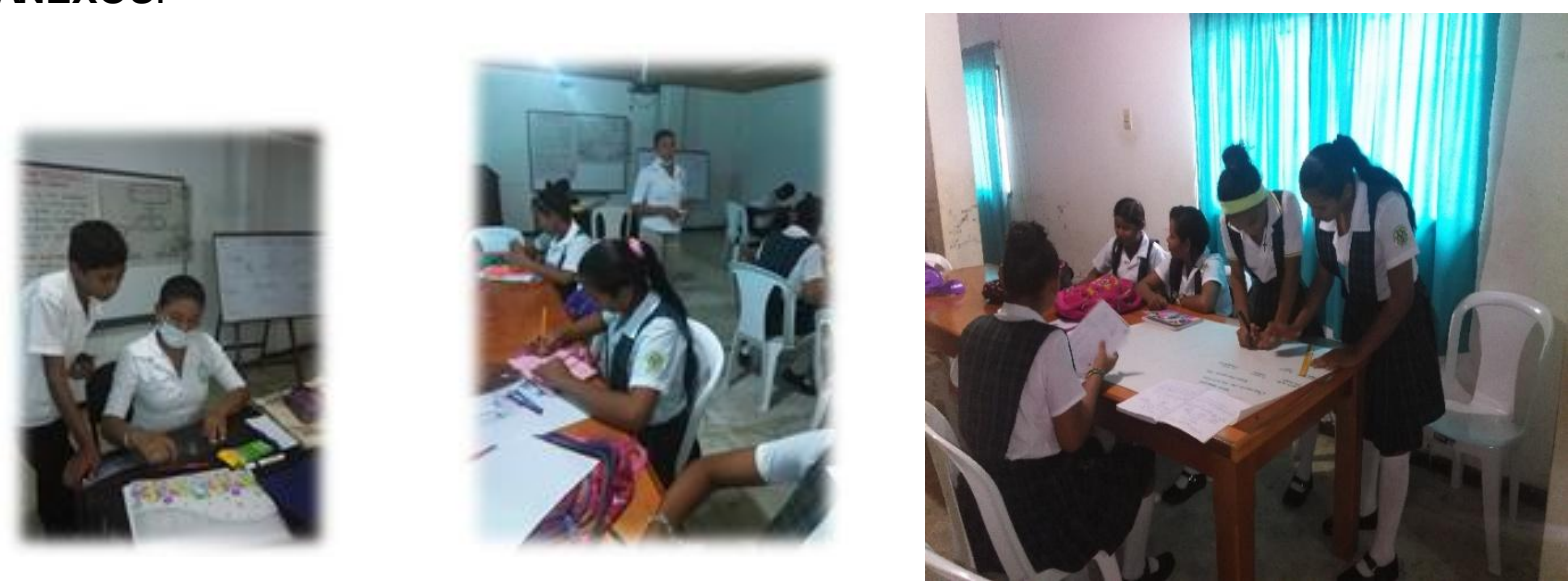